\title{
Thermal Conductivity of Epoxy Resin Reinforced with Magnesium Oxide Coated Multiwalled Carbon Nanotubes
}

\author{
Fei-Peng Du, Hao Tang, and De-Yong Huang \\ School of Materials Science and Engineering, Wuhan Institute of Technology, Wuhan 430073, China \\ Correspondence should be addressed to Fei-Peng Du; hsdfp@163.com
}

Received 9 June 2013; Accepted 2 August 2013

Academic Editor: Yingkui Yang

Copyright (C) 2013 Fei-Peng Du et al. This is an open access article distributed under the Creative Commons Attribution License, which permits unrestricted use, distribution, and reproduction in any medium, provided the original work is properly cited.

\begin{abstract}
Magnesium oxide coated multiwalled carbon nanotubes (MgO@MWNT) were fabricated and dispersed into epoxy matrix. The microstructures of MgO@MWNT and epoxy/MgO@MWNT nanocomposites were characterized by TEM and SEM. Electrical resistivity and thermal conductivity of epoxy nanocomposites were investigated with high resistance meter and thermal conductivity meter, respectively. MgO@MWNT has core-shell structure with MgO as shell and nanotube as core, and the thickness of $\mathrm{MgO}$ shell is ca.15 nm. MgO@MWNT has been dispersed well in the epoxy matrix.MgO@MWNT loaded epoxy nanocomposites still retain electrical insulation inspite of the filler content increase. However, thermal conductivity of epoxy was increased with the MgO@MWNT content increasing. When MgO@MWNT content reached 2.0 wt.\%, thermal conductivity was increased by 89\% compared to neat epoxy, higher than that of unmodified MWNT nanocomposites with the same loading content.
\end{abstract}

\section{Introduction}

Integrated circuit with high integration and miniaturization has resulted in a large amount of waste heat that is produced when electronic components work at high frequency. Accumulated heat should make semiconductors' working stability poor and life expectancy short under higher thermal environment [1]. High performance of electronic packaging material is expected to transport the heat to the integrated circuit [2]. Traditionally, some microsize fillers with high thermal conductivity have been added into polymer matrix to obtain electronic packaging materials [3-5]. However, high content of filler makes mechanical properties of composites deteriorate.

Carbon nanotubes (CNT) have ultrahigh thermal conductivity, such as single-walled carbon nanotubes (SWNT) with $6000 \mathrm{~W} /(\mathrm{m} \cdot \mathrm{k})$ and multiwalled carbon nanotubes (MWNT) with $3000 \mathrm{~W} /(\mathrm{m} \cdot \mathrm{k})$ [6, 7]. Theoretically, small amount of CNT added can sharply improve the thermal conductivity of polymer matrix [8]. In fact, thermal conductivity improved by CNT is very far below the expected value, which results from the interface thermal resistance between $\mathrm{CNT}$ and polymer [9]. The interface thermal resistance weakened the heat flow transporting in the composites. To decrease thermal resistance and enhance heat flow transporting in the interface of CNT and polymer, some researches focused on the interface design between carbon nanomaterial and polymer to improve the thermal conductivity or other properties of polymer composites [10-14]. Although CNTs can improve the thermal conductivity of polymer, their excellent electrical conductive property can significantly change the electric conductivity of the polymer matrix. So, polymer/CNT composites are hardly appropriate as electronic packaging materials or thermal interface materials $[15,16]$. To overcome this issue, coaxial cable structure of CNT coated with hyper-branched polymer or nanosize silica was added into polymer matrix via interface design method in our previous work $[15,16]$. This kind of electrical-insulating layer between polymer and CNT can restrain electronic transport properties of CNT in the composites and keep the polymer composite electrical insulation.

To obtain high thermal conductivity of polymer composites, some researches should be done to investigate the influence of different interface layers on thermal conductivity. 


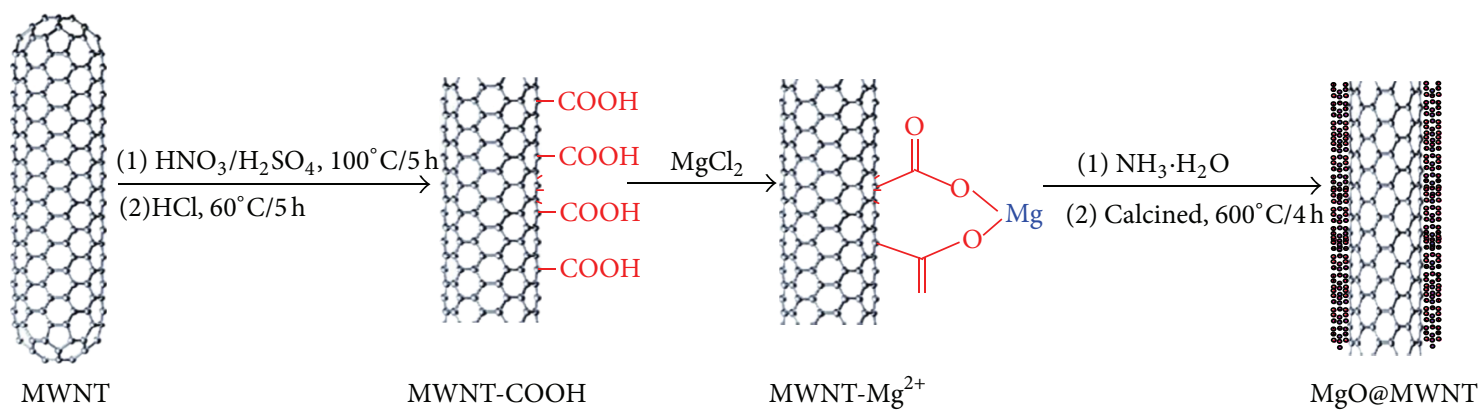

FIGURE 1: Fabrication scheme of MgO@MWNT hybrid.

In this paper, epoxy/MWNT nanocomposites have been prepared via coating magnesium oxide $(\mathrm{MgO})$ on the surface of MWNT. The influence of thermal conductivity of interface layer on the composite material has been investigated.

\section{Experimental}

2.1. Materials. Raw multi-walled carbon nanotubes ( $r-$ MWNTs) were purchased from Shenzhen Nano Harbor Co,. (China), with the diameter of 20-40 $\mathrm{nm}$ and $95 \%$ purity. Diglycidyl ether of biphenol A (E51) was purchased from Shanghai Resin Factory (China). 2-ethyl-4-methylimidazole was purchased from Xian Hangang Chemical Co., Ltd. (China). All other reagents were analytical reagents and purchased from Sinopharm Chemical Reagent Co., Ltd (China).

2.2. Purification of MWNT. The purification of MWNT can be referred to in some documents [17]. The typical process was as follows: $2 \mathrm{~g}$ MWNT was added into the $200 \mathrm{~mL}$ mixture of $\mathrm{H}_{2} \mathrm{SO}_{4}$ and $\mathrm{HNO}_{3}(\mathrm{v} / \mathrm{v}=1: 3)$ with ultrasound treating and refluxing at $100^{\circ} \mathrm{C}$ for $5 \mathrm{~h}$. After finishing the reaction, the oxidized $\mathrm{MWNT}$ was washed several times with deionized water via vacuum filtering with $0.22 \mu \mathrm{m}$ pore size of nylon membrane and then dried in the vacuum oven at $60^{\circ} \mathrm{C}$. The oxidized MWNT was further added into $\mathrm{HCl}$ concentrated solution at $60^{\circ} \mathrm{C}$ for $6 \mathrm{~h}$ and then washed with deionized water via vacuum filtering to obtain pure oxidized MWNT (o-MWNT).

2.3. Preparation of Magnesium Oxide Coated MWNT Hybrid (MgO@MWNT).100 mg o-MWNT was dispersed in ethanol with ultrasonic treating. The obtained MWNT suspension was added to $25 \mathrm{~mL} \mathrm{MgCl}_{2}$ solution $(0.1 \mathrm{M})$ under magnetic stirring and kept stirring for $30 \mathrm{~min}$, and the weight ration of CNT to $\mathrm{MgO}$ can reach 1:1. After that, the mixture was slowly added with ammonia solution at $50^{\circ} \mathrm{C}$ in order to turn $\mathrm{Mg}^{2+}$ into $\mathrm{Mg}(\mathrm{OH})_{2}$ under alkaline condition. After keeping the reaction for $2 \mathrm{~h}$, the mixture was set aside at room temperature for $2 \mathrm{~h}$. And then, the mixture was filtered, washed with water and ethanol repeatedly until there were no ions in the filter liquor, and dried at $80^{\circ} \mathrm{C}$ in the vacuum oven to obtain the precursor of MgO@MWNT. Finally, the precursor was calcined in argon atmosphere at $600^{\circ} \mathrm{C}$ for $4 \mathrm{~h}$ to obtain MgO@MWNT.
2.4. Preparation of Epoxy/MgO@MWNT Nanocomposites. Above all,MgO@MWNT and a certain content of epoxy were added into acetone and ultrasonically dispersed at $35^{\circ} \mathrm{C}$ for $30 \mathrm{~min}$. And then, hexahydrophthalic anhydride as curing agent was poured into the mixture solution with mechanical stirring and ultrasonic treating simultaneously at $35^{\circ} \mathrm{C}$ for $45 \mathrm{~min}$, so that all components were mixed uniformly. Furthermore, the mixture was poured into the disk mould coated with demould agent to extract acetone and remove bubbles in vacuum oven at $60^{\circ} \mathrm{C}$ for $2 \mathrm{~h}$. Finally, the mixture was kept at $80^{\circ} \mathrm{C}$ for $2 \mathrm{~h}$, heated to $140^{\circ} \mathrm{C}$ and kept the temperature for $2 \mathrm{~h}$, and then naturally cooled to room temperature in turn. Epoxy/MgO@MWNT composites were obtained after demoulding.

2.5. Characterization. The morphology of MgO@MWNT and the microstructure of epoxy/MgO@MWNT were examined by Tecnai G220 electron microscope (FEI Co., The Netherlands) and Sirion 200 field emission scanning electron microscope (FEI Co., The Netherlands). Thermal conductivity of the composites was measured with QTM-500 quick thermal conductivity meter (KEM Co., Japan). Volume electrical resistivity measurements were conducted using a plate electrode type ZC36 high resistance meter (Shanghai Cany Precision Instrument, Co., China). And the surfaces of samples were polished with sandpaper before measuring.

\section{Results and Discussion}

3.1. Functionalization of Multiwalled Carbon Nanotubes. As shown in Figure 1, MWNT was first oxidized with a mixture of concentrated sulfuric acid and nitric acid to get rid of impurity and obtain carboxylic acid groups on the surface of nanotubes [17]. The carboxylic acid groups have strong interaction with metal ion due to chelating, so that metal ion can be absorbed on the surface of nanotubes. After adding ammonia, $\mathrm{Mg}^{2+}$ was transferred into insoluble $\mathrm{Mg}(\mathrm{OH})_{2}$ and then calcined to $\mathrm{MgO}$ coated on the surface of nanotubes. Based on the gravitational thermal analysis, the content of $\mathrm{MgO}$ in MgO@MWNT hybrid is ca. 50 wt.\%.

$\mathrm{MgO}$ coated on the surface of nanotubes can be investigated with SEM and TEM observations. As previously observed, no impurity or agraphitic carbon was observed among o-MWNT after acidic treating of r-MWNT [17]. 


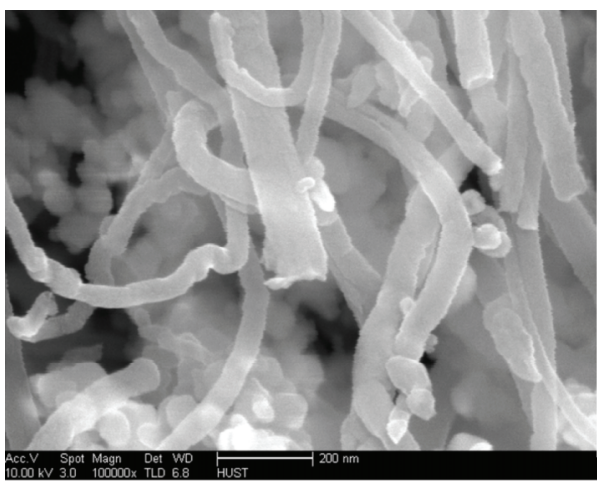

(a)

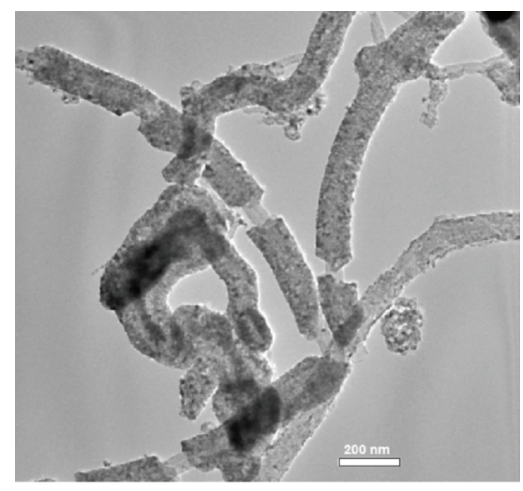

(b)

FIGURE 2: (a) SEM image and (b) TEM image of MgO@MWNT hybrid.

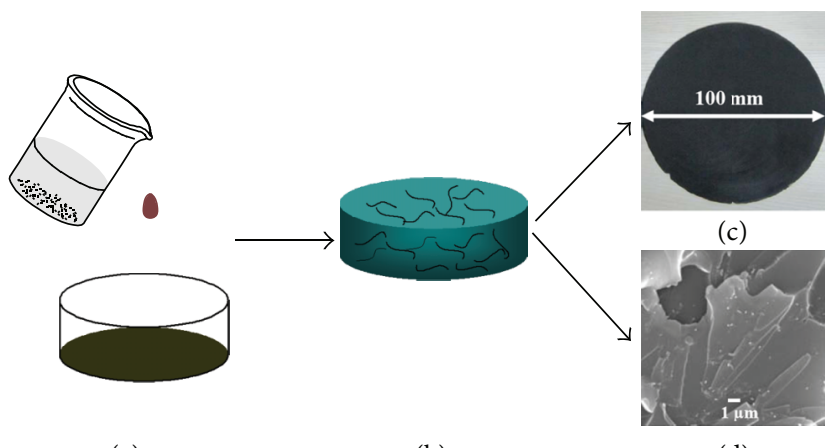

(a)

(b)

(d)

FIGURE 3: Scheme of (a) the mixture of epoxy and MgO@MWNT poured into mould and (b) the sample of epoxy/MgO@MWNT nanocomposites, (c) the sample photograph of epoxy/MgO@MWNT nanocomposite, and (d) SEM image of cross-section for epoxy/MgO@MWNT nanocomposites.

As shown in Figure 2(a), the surface of carbon nanotubes showed the coarse and granular structure. And some free particles were found among carbon nanotubes without coating on the surface of nanotubes. TEM image in Figure 2(b) clearly showed that the nanotubes were coated with $\mathrm{MgO}$, and a concentric cable structure was formed with nanotube as core and $\mathrm{MgO}$ as shell similar with other hybrid structures of carbon nanotubes [16]. From the observation of TEM images, the thickness of $\mathrm{MgO}$ coating is ca. $15 \mathrm{~nm}$. The coatings of $\mathrm{MgO}$ have some cracks caused by calcination.

\subsection{Microstructure of Epoxy/MgO@MWNT Nanocompos-} ites. The simple fabrication process of epoxy/MgO@MWNT nanocomposites and the dispersion of $\mathrm{MgO} @ \mathrm{MWNT}$ in the polymer matrix are shown in Figure 3 . The mixture of MWNT and epoxy in acetone solvent is easily treated under ultrasound treating without a precipitate in the bottom of the vessel, and the mixture is easily leveled in the mould. The photograph of the sample shows that the surfaces of epoxy/MgO@MWNT are wafer smooth (Figure 3(c)). The SEM image shows that MgO@MWNT are uniformly dispersed into epoxy matrix after curing (Figure 3(d)). The previous work showed that r-MWNT are aggregated together in polymer matrix due to their incompatibility [16]. The good dispersion of $\mathrm{MgO} @ \mathrm{MWNT}$ is attributed to the hydroxyl groups of $\mathrm{MgO}$ layer on the surface of MWNT. So, MgO@MWNT has a good compatibility with epoxy matrix, and the $\mathrm{MgO}$ layer prevents the nanotubes aggregation and bundle formation.

3.3. Electrical Conductivity of Epoxy/MgO@MWNT Nanocomposites. The volume electrical conductivity of epoxy/MWNT nanocomposites can be shown in Figure 4. The $r-$ MWNT has excellent electrical conductivity and has been used to improve the electrical conductivity of polymer matrix. And only a very low content of MWNT can obviously improve the electrical conductivity of polymer. Here, neat epoxy is in electrical-insulating state with $3.8 \times 10^{15} \Omega \cdot \mathrm{m}$ of electrical resistivity at room temperature. The electrical conductivity of epoxy nanocomposites is increasing with the content increase of r-MWNT. When r-MWNT content is only $0.5 \mathrm{wt} . \%$, the electrical resistivity of nanocomposite sharply decreases by 6 orders of magnitude compared to neat epoxy. With further loading r-MWNT from $1 \mathrm{wt} . \%$ to $4.0 \mathrm{wt} . \%$, electrical resistivity of the nanocomposites decreases mildly from $6.4 \times 10^{8} \Omega \cdot \mathrm{m}$ to $2.0 \times 10^{7} \Omega \cdot \mathrm{m}$. Therefore, the sharp change of electrical resistivity indicated that the electricalpercolating network was already formed with CNT loading no more than $0.5 \mathrm{wt} . \%$. 


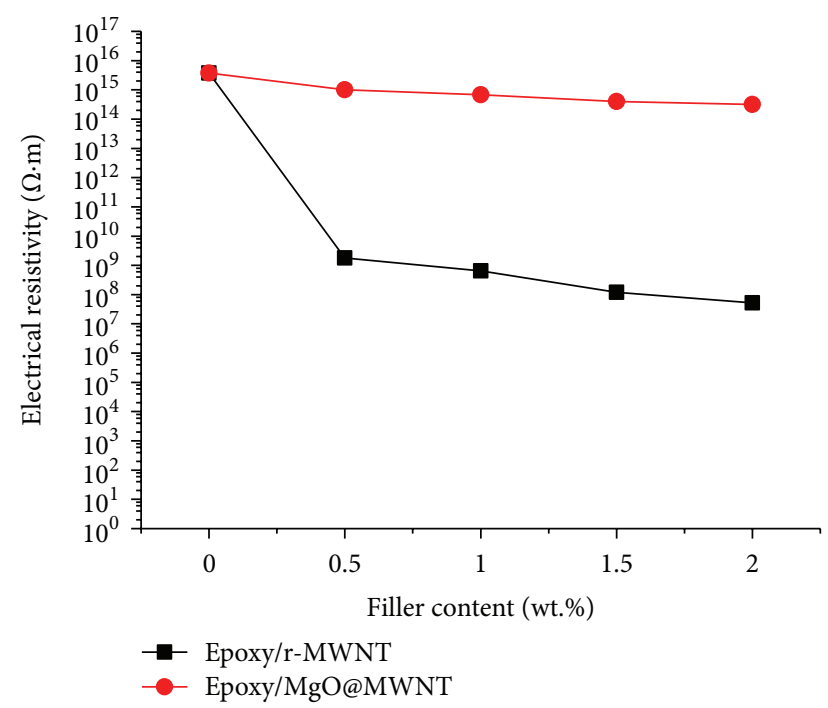

FIGURE 4: Electrical resistivity of epoxy nanocomposites filled with r-MWNT and MgO@MWNT.

However, it is obvious that epoxy/MgO@MWNT nanocomposites hold different electrical properties compared with epoxy/r-MWNT nanocomposites as shown in Figure 4. With the content increasing of MgO@MWNT from 0.5 wt.\% to $4.0 \mathrm{wt} . \%$, electrical resistivity of the nanocomposites has little change of order and is almost equal to that of neat epoxy. With 4 wt.\% MgO@MWNT loading, electrical resistivity of epoxy/MgO@MWNT nanocomposites only decreases to 2.4 $\times 10^{14} \Omega \cdot \mathrm{m}$ from $3.8 \times 10^{15} \Omega \cdot \mathrm{m}$ of neat epoxy. Therefore, the obtained results indicated that the $\mathrm{MgO}$ shell layer on the surface of nanotubes has the importance to bring epoxy/MgO@MWNT nanocomposites electrical insulation. The electrical-insulating coating can restrain electron/charge transport on the interfacial and intertubes $[18,19]$. $\mathrm{MgO}$ is an electrical-insulating coating on the surface of MWNT, so that epoxy/MgO@MWNT nanocomposites maintain the electrical insulation as the neat epoxy. The similar results were obtained in our previous researches $[15,16]$ or other documents [20,21].

3.4. Thermal Conductivity of Epoxy/MgO@MWNT Nanocomposites. The thermal conductivity is shown in Figure 5 for epoxy nanocomposites with different content of MWNT loading. The thermal conductivity of neat epoxy is $0.1932 \mathrm{~W} /$ $(\mathrm{m} \cdot \mathrm{K})$. The thermal conductivity of epoxy/r-MWNT nanocomposites increases with r-MWNT content increasing. Compared to neat epoxy, the thermal conductivity of epoxy nanocomposites with the r-MWNT of $0.5 \mathrm{wt} . \%, 1.0 \mathrm{wt} . \%$, $1.5 \mathrm{wt} . \%$, and $2.0 \mathrm{wt} . \%$ was increased by $15 \%, 38 \%, 43 \%$, and $50 \%$, respectively. The epoxy nanocomposites with 2.0 wt.\% r-MWNT loaded have the thermal conductivity of $0.2898 \mathrm{~W} /(\mathrm{m} \cdot \mathrm{K})$. However, it is clearly observed that epoxy/MgO@MWNT nanocomposites exhibit higher thermal conductivity than epoxy/r-MWNT nanocomposites. Correspondingly, with the increase of MgO@MWNT content from $0.5 \mathrm{wt} . \%$ to $2.0 \mathrm{wt} . \%$, the thermal conductivity of epoxy

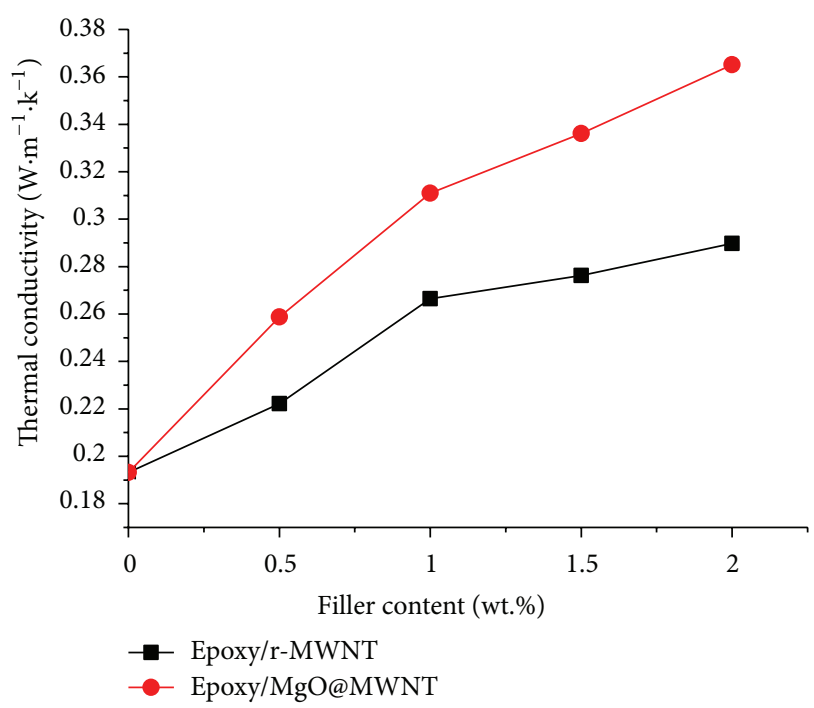

FIgURE 5: Thermal conductivity of epoxy nanocomposites filled with r-MWNT and MgO@MWNT.

nanocomposites was increased from $34 \%$ to $89 \%$. The thermal conductivity of 2.0 wt.\% MgO@MWNT loaded epoxy reached $0.3568 \mathrm{~W} /(\mathrm{m} \cdot \mathrm{K})$.

Our previous researches showed that the polymer containing hydroxyl group or silica coated on the surface of MWNT can obviously improve the interfacial thermal conductivity of polymer nanocomposites $[15,16]$. On one hand, the hydroxyl groups of inorganic or organic coating have strong interaction with epoxy matrix which can promote phonon propagation through the interfaces of composites. On the other hand, the coatings on the surface of MWNT can be used as transition layer, and their modulus values are between rigid carbon nanotubes and soft polymer matrix, so that the coating can decrease the acoustic impedance mismatch and increase the interfacial thermal transport. $\mathrm{MgO}$ coating has the same function to improve the interfacial thermal conduction of epoxy/MWNT nanocomposites. The results further confirmed that suitable coating on the surface of carbon nanotubes can decrease the phonon scattering on the interface of carbon nanotubes and polymer.

The mechanism of thermal conduction and electrical conduction is different in the epoxy nanocomposites with MWNT loading. The thermal conduction depends on the phonon movement but the electrical conduction depends on electron transport. The electrical-insulating layer can shield electron transport but could not hinder the phonon propagation. So, MWNT coated with a suitable electricalinsulating layer can improve thermal conductivity and retain the high electrical resistivity of epoxy resin simultaneously.

\section{Conclusions}

MgO coated MWNT was successfully fabricated and formed a shell-core structure with ca. $15 \mathrm{~nm}$ thickness of $\mathrm{MgO}$ as shell and nanotubes as core. MgO@MWNT was well dispersed in the epoxy matrix due to good compatibility between 
nanotubes and epoxy matrix. The electrical-insulating layer of $\mathrm{MgO}$ restrained the electron transport properties of carbon nanotubes and kept epoxy/MgO@MWNT nanocomposites electrical insulation. The $\mathrm{MgO}$ layer also promoted nanotube strong interaction with epoxy matrix and matched the modulus of MWNT and polymer matrix that improved the interfacial thermal conductivity. The thermal conductivity of epoxy/MgO@MWNT nanocomposites is obviously higher than that of epoxy/r-MWNT nanocomposites with the same filler content. High electrical insulation and thermal conductivity will make epoxy/MgO@MWNT nanocomposites promising in the electrical package field.

\section{Acknowledgment}

This work was supported by Young Fund of the National Natural Science Foundation of China (50903034).

\section{References}

[1] P. Y. Paik, V. K. Pamula, and K. Chakrabarty, "Adaptive cooling of integrated circuits using digital microfluidics," IEEE Transactions on Very Large Scale Integration (VLSI) Systems, vol. 16, no. 4, pp. 432-443, 2008.

[2] A. M. Abyzov, S. V. Kidalov, and F. M. Shakhov, "High thermal conductivity composite of diamond particles with tungsten coating in a copper matrix for heat sink application," Applied Thermal Engineering, vol. 48, no. 15, pp. 72-80, 2012.

[3] B. L. Zhu, J. Ma, J. Wu, K. C. Yung, and C. S. Xie, "Study on the properties of the epoxy-matrix composites filled with thermally conductive AlN and BN ceramic particles," Journal of Applied Polymer Science, vol. 118, no. 5, pp. 2754-2764, 2010.

[4] H. He, R. Fu, Y. Han, Y. Shen, and D. Wang, "High thermal conductive $\mathrm{Si}_{3} \mathrm{~N}_{4}$ particle filled epoxy composites with a novel structure," Journal of Electronic Packaging, vol. 129, no. 4, pp. 469-472, 2007.

[5] X. C. Tong, K. Wattanakul, H. Manuspiya, and N. Yanumet, "Thermal conductivity and mechanical properties of BN-filled epoxy composite: effects of filler content, mixing conditions, and BN agglomerate size," Journal of Composite Materials, vol. 45, no. 19, pp. 1967-1980, 2011.

[6] S. Berber, Y. Kwon, and D. Tománek, "Unusually high thermal conductivity of carbon nanotubes," Physical Review Letters, vol. 84, no. 20, pp. 4613-4616, 2000.

[7] P. Kim, L. Shi, A. Majumdar, and P. L. McEuen, "Thermal transport measurements of individual multiwalled nanotubes," Physical Review Letters, vol. 87, no. 21, Article ID 215502, 4 pages, 2001.

[8] J. E. Peters, D. V. Papavassiliou, and B. P. Grady, "Unique thermal conductivity behavior of single-walled carbon nanotubepolystyrene composites," Macromolecules, vol. 41, no. 20, pp. 7274-7277, 2008.

[9] S. T. Huxtable, D. G. Cahill, S. Shenogin et al., "Interfacial heat flow in carbon nanotube suspensions," Nature Materials, vol. 2, no. 11, pp. 731-734, 2003.

[10] S. Shenogin, A. Bodapati, L. Xue, R. Ozisik, and P. Keblinski, "Effect of chemical functionalization on thermal transport of carbon nanotube composites," Applied Physics Letters, vol. 85, no. 12, pp. 2229-2231, 2004.
[11] S. Yang, C. M. Ma, C. Teng et al., "Effect of functionalized carbon nanotubes on the thermal conductivity of epoxy composites," Carbon, vol. 48, no. 3, pp. 592-603, 2010.

[12] Y. K. Yang, L. J. Yu, R. G. Peng et al., "Incorporation of liquidlike multiwalled carbon nanotubes into an epoxy matrix by solvent-free processing," Nanotechnology, vol. 23, no. 22, Article ID 225701, 2012.

[13] Y. Yang, C. He, R. Peng et al., "Non-covalently modified graphene sheets by imidazolium ionic liquids for multifunctional polymer nanocomposites," Journal of Materials Chemistry, vol. 22, no. 12, pp. 5666-5675, 2012.

[14] Y. Yang, X. Xie, Z. Yang et al., "Controlled synthesis and novel solution rheology of hyperbranched poly(ureaurethang)-functionalized multiwalled carbon nanotubes," Macromolecules, vol. 40, no. 16, pp. 5858-5867, 2007.

[15] J. Zhao, F. Du, X. Zhou et al., "Thermal conductive and electrical properties of polyurethane/hyperbranched poly(ureaurethane)-grafted multi-walled carbon nanotube composites," Composites B, vol. 42, no. 8, pp. 2111-2116, 2011.

[16] W. Cui, F. Du, J. Zhao et al., "Improving thermal conductivity while retaining high electrical resistivity of epoxy composites by incorporating silica-coated multi-walled carbon nanotubes," Carbon, vol. 49, no. 2, pp. 495-500, 2011.

[17] F. Du, K. Wu, Y. Yang, L. Liu, T. Gan, and X. Xie, "Synthesis and electrochemical probing of water-soluble poly(sodium 4styrenesulfonate-co-acrylic acid)-grafted multiwalled carbon nanotubes," Nanotechnology, vol. 19, no. 8, Article ID 085716, 2008.

[18] F. M. Blighe, Y. R. Hernandez, W. J. Blau, and J. N. Coleman, "Observation of percolation-like scaling-far from the percolation threshold-in high volume fraction, high conductivity polymer-nanotube composite films," Advanced Materials, vol. 19, no. 24, pp. 4443-4447, 2007.

[19] B. E. Kilbride, J. N. Coleman, J. Fraysse et al., "Experimental observation of scaling laws for alternating current and direct current conductivity in polymer-carbon nanotube composite thin films," Journal of Applied Physics, vol. 92, no. 7, pp. 40244030, 2002.

[20] C. Velasco-Santos, A. L. Martínez-Hernández, M. LozadaCassou, A. Alvarez-Castillo, and V. M. Castaño, "Chemical functionalization of carbon nanotubes through an organosilane," Nanotechnology, vol. 13, no. 4, pp. 495-498, 2002.

[21] P. C. Ma, J. Kim, and B. Z. Tang, "Effects of silane functionalization on the properties of carbon nanotube/epoxy nanocomposites," Composites Science and Technology, vol. 67, no. 14, pp. 2965-2972, 2007. 

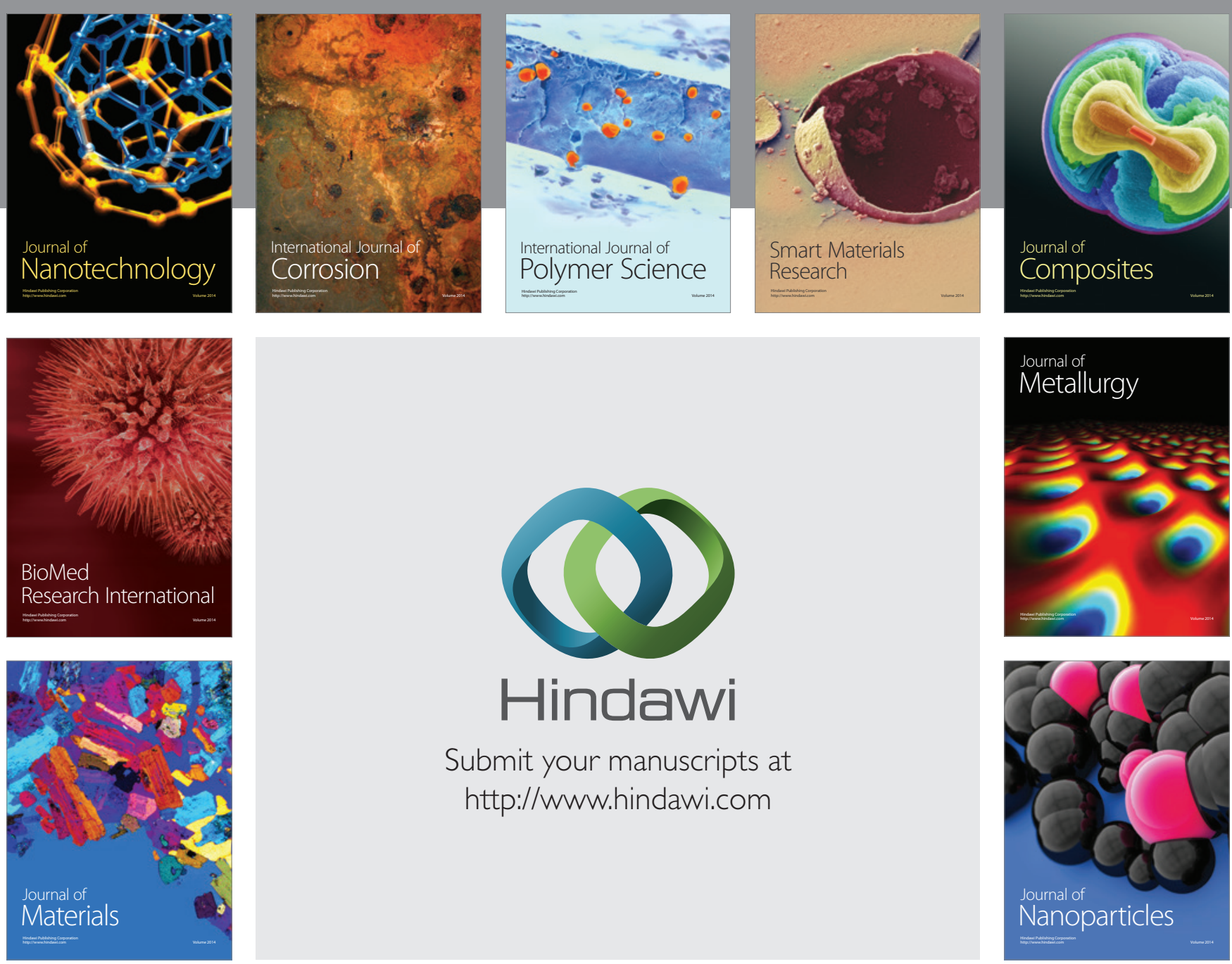

Submit your manuscripts at http://www.hindawi.com
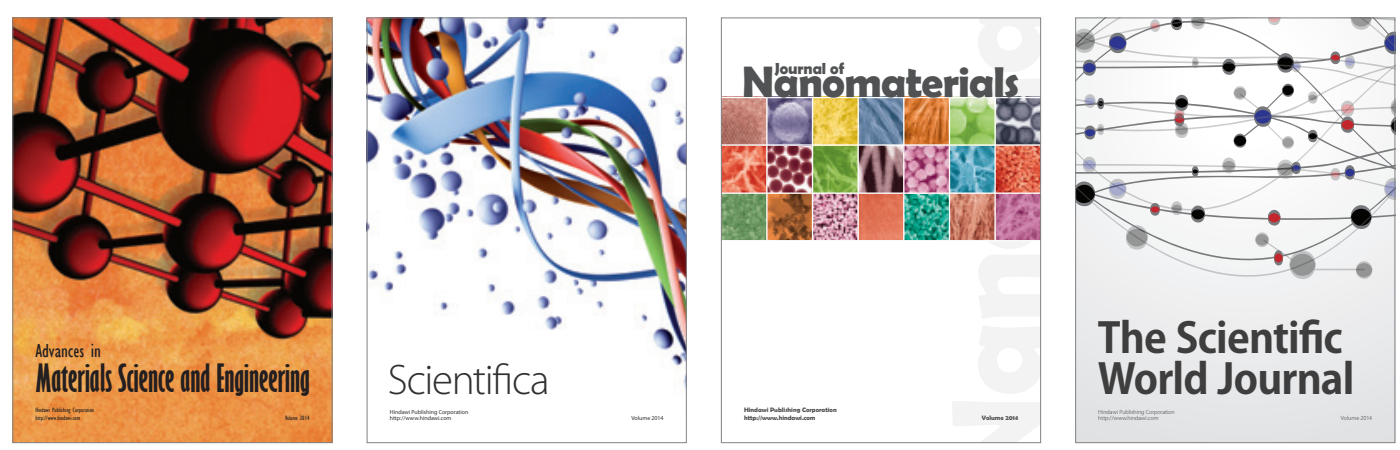

\section{The Scientific World Journal}
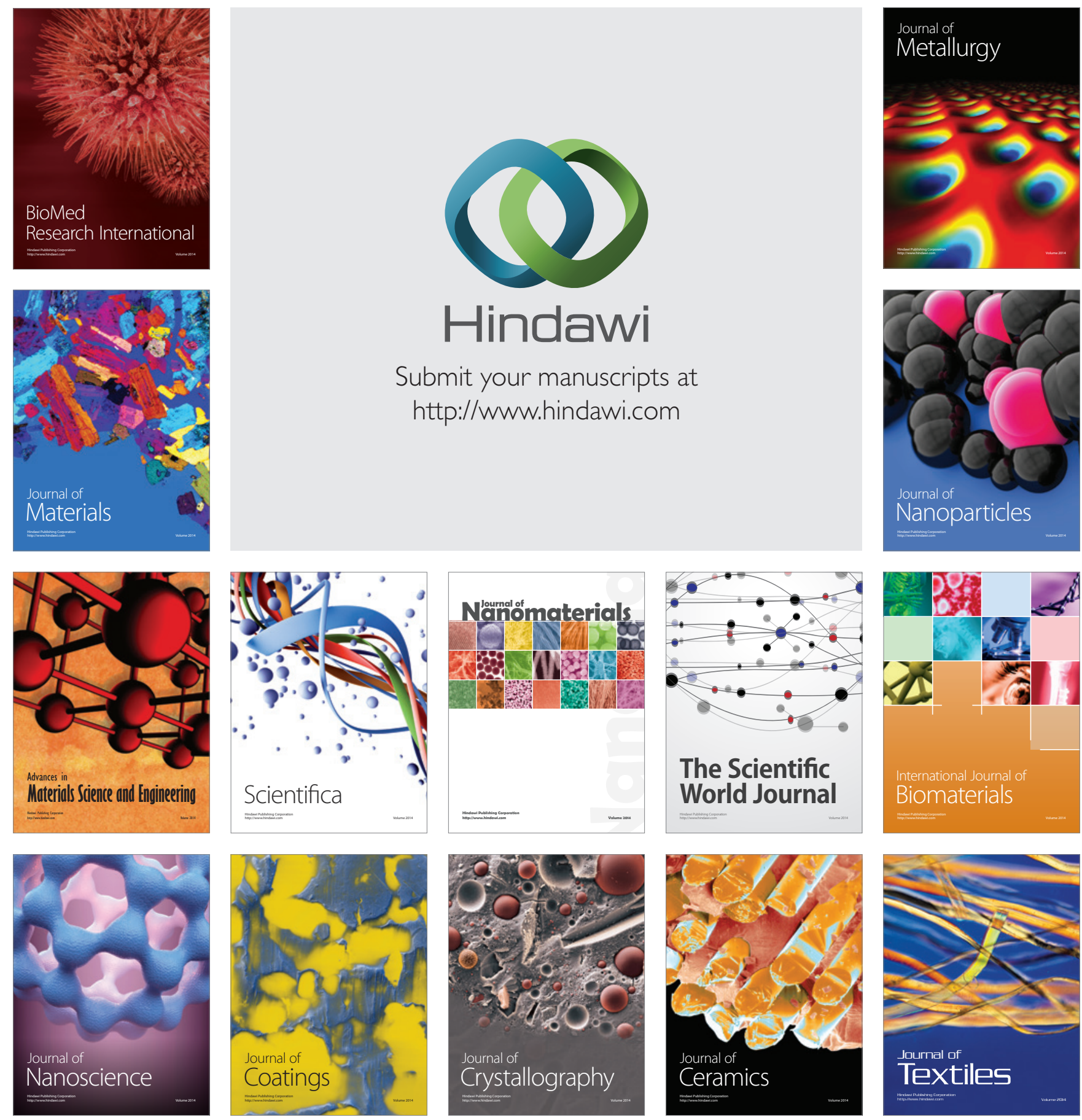